\title{
Light-Scattering Spectroscopy
}

National Cancer Institute

\section{Source}

National Cancer Institute. Light-Scattering Spectroscopy. NCI Thesaurus. Code C20059.

The nuclei of precancerous lesions are distinct in that they become enlarged, crowded and hyperchromatic. Light-scattering spectroscopy can diagnose early epithelial cancers based on the scattering of light through their enlarged nuclei. (from Medscape) 